\title{
POLÍTICA NACIONAL DE PROMOÇÃO DA SAÚDE E POLÍTICA NACIONAL DO ESPORTE: UM VÍNCULO NECESSÁRIO
}

\author{
NATIONAL HEALTH PROMOTION POLICY AND NATIONAL \\ SPORTS POLICY: A NECESSARY LINK
}

Agnes Ramos Guirelli (Orcid: 0000-0001-5852-7823) 1 Ana Luíza Costa e Silva Cabral (Orcid: 0000-0002-0172-5957) 1 Edneia de Oliveira Salum (Orcid: 0000-0003-2342-9400) 1 Marilita Falangola Accioly (Orcid: 0000-0002-9623-3145) 1,2 Adele Ferreira dos Santos (Orcid: 0000-0002-2253-3196) 1 Dernival Bertoncello (Orcid: 0000-0002-4432-4651) 1,2 Isabel Aparecida Porcatti de Walsh (Orcid: 0000-0002-2317-1326) 1,2

Autor correspondente:

Isabel Aparecida Porcatti de Walsh

E-mail: isabelpwalsh@gmail.com

${ }^{1}$ Programa de Pós-Graduação em Fisioterapia. Universidade Federal do Triângulo Mineiro e Universidade Federal de Uberlândia

Departamento de Fisioterapia Aplicada. Universidade Federal do Triângulo Mineiro

\section{RESUMO}

A Política Nacional de Promoção da Saúde (PNPS) e a Política Nacional do Esporte (PNE) enfrentam dificuldades para a inclusão social, sobre falta de conhecimento dos gestores, escassez de desenvolvimento técnico-científico, carência de investimentos governamentais, mas, principalmente, grande necessidade de implementação de ações eficazes das duas partes. Com todas as dificuldades enfrentadas pela PNPS no que diz respeito à implementação eficaz de suas ações, é visto que o esporte, no âmbito educacional e de participação, tem tido investimentos escassos por parte do governo federal, contrariando as premissas de inclusão social e de incentivo à saúde e lazer. Tendo a saúde como um componente central dessas políticas, faz-se essencial destacar a importância de fortalecer a articulação das políticas nacionais. Vários preceitos devem ser revistos e destacados para melhorar e ampliar a PNE e suas estratégias de implementação, levando em consideração a importância de fortalecer a articulação de políticas públicas como a PNPS e a PNE, que têm objetivos e diretrizes semelhantes, visando elaborar estratégias mais eficazes no que diz respeito à prática de atividade física regular e saúde da população em geral. Ainda, cabe aos profissionais da saúde atuar em ações intersetoriais, inter e transdisciplinares, pretendendo a integralidade da assistência. É importante alinhar as políticas e utilizar o esporte recreativo como estratégia de promoção da saúde, por meio de ações que abranjam os dois objetivos: promoção da saúde e democratização do esporte.

PALAVRAS-CHAVE: Promoção da saúde; Esportes; Política pública; Colaboração intersetorial.

\begin{abstract}
The National Health Promotion Policy (NHPP), together with the National Sports Policy (NEP), face difficulties that lead to social inclusion, lack of managerial knowledge, lack of technical and scientific development, lack of government investments, but mainly a great need for implementation of effective actions of both policies. With all the difficulties faced by the NHPP regarding the effective implementation of its actions, it is seen that sports in the educational and participation scope have had scarce investments by the federal government, contrary to the premises of social inclusion. With health as a central component of those policies, it is essential to stress the importance of strengthening the articulation of the national policies in question. Several precepts should be reviewed and highlighted to improve and broaden NEP and its implementation strategies, taking into account the importance of strengthening the articulation of public policies such as NHPP and NEP, which have similar objectives and guidelines, with the aim of elaborating more effective strategies in relation to the physical activity and health of the population in general. Moreover, it is the responsibility of health professionals to act in intersectoral, inter, and transdisciplinary actions, aiming at the integrality of care. It is important to align policies and use recreational sports as a health promotion strategy, through actions that address the two goals: health promotion and the democratization of sports.
\end{abstract}




\section{ATIVIDADE FÍSICA COMO PROMOTORA DE SAÚDE}

Estudos epidemiológicos demonstram que a inatividade física aumenta substancialmente a incidência relativa de doença arterial coronariana (45\%), infarto agudo do miocárdio (60\%), hipertensão arterial (30\%), câncer de cólon (41\%), câncer de mama (31\%), diabetes do tipo II (50\%) e osteoporose $(59 \%)^{1}$.

Já a prática de Atividade Física (AF) gera melhoria da qualidade de vida, da mobilidade e da capacidade funcional; e, em indivíduos idosos, os benefícios se mostram mais relevantes devido às alterações que o próprio processo de senescência ocasiona $^{2-4}$. Pesquisas mostram também benefícios que a prática esportiva e das AF ocasionam na saúde mental nos aspectos autoestima, depressão, ansiedade, insônia, além da melhoria na memória, aprendizagem, atenção, estando associada com o menor risco de demência, demência senil e doença de Alzheimer².

São muitas as possibilidades que contemplam o movimento humano em diferentes modalidades (exercício, ginástica, jogo, esporte, luta/arte marcial, dança, recreação) e perspectivas (prevenção, promoção, proteção e reabilitação da saúde; formação cultural; educação e reeducação motora; rendimento físico-esportivo; lazer; gestão de empreendimentos relacionados a estas temáticas; dentre outros $)^{5}$. No entanto, estudos recentes demonstram que grande parcela da população brasileira não pratica AF como o recomendado ${ }^{6}$. O sedentarismo vem sendo um problema de saúde pública, justificando a importância de ações voltadas para estimular a prática de $\mathrm{AF}$, ampliando as ações das políticas públicas direcionadas a essa área.

\section{POLÍTICA NACIONAL DE PROMOÇãO DA SAÚDE}

A Política Nacional de Promoção da Saúde (PNPS) foi instituída pela Portaria MS/GM n ${ }^{\circ}$ 687 , de 30 de março de 2006, e redefinida pela Portaria $\mathrm{n}^{\circ} 2.446$, de 11 de novembro de 2014, que foi revogada pela Portaria de Consolidação $\mathrm{n}^{\circ} 2$, de 28 de setembro de 2017, que consolidou as normas das políticas nacionais de saúde do Sistema Único de Saúde (SUS), definindo o compromisso que o Estado brasileiro tem com as ações de promoção da saúde nos serviços e na gestão do SUS 7 .

A PNPS traz o conceito ampliado de saúde como um conjunto de estratégias e formas de gerar saúde, no âmbito individual e coletivo. Seus objetivos, princípios e diretrizes visam à equidade, à melhoria das condições e dos modos de viver e à afirmação do direito à vida e à saúde, entendendo que o conceito e as ações em promoção de saúde são mais abrangentes do que ações voltadas apenas para prevenção de doenças $^{8}$. Essa Política definiu como uma de suas prioridades a $\mathrm{AF}$ e as práticas corporais.

A Portaria no 719, de 7 de Abril de 2011, institui o Programa Academia da Saúde, com objetivo principal de contribuir para a promoção da saúde da população ${ }^{9}$, visando, principalmente, à promoção de práticas corporais e atividades físicas com a implantação de polos com infraestrutura e equipamentos direcionados à prática de atividades individuais e coletivas com orientação de profissionais qualificados, bem como seu custeio, prevendo ainda o desenvolvimento de atividades de segurança alimentar, nutrição e outros modos de vida saudável ${ }^{9}$. Este foi redefinido em 2013, com seu objetivo ampliado para contribuir para a promoção da saúde e produção do cuidado e de modos de vida saudáveis da população Brasil ${ }^{10}$. 
Uma das formas apontadas pela própria PNPS revisada, para seu fortalecimento, é a de se articular com outras políticas públicas, visto que é mais fácil alcançar os objetivos e metas quanto todos os setores incorporam a saúde como um tema central. Além do compartilhamento de metas, é de grande importância econômica o compartilhamento de recursos físicos e humanos ${ }^{7}$. Assim, destaca-se a relevância da articulação da PNPS com a Política Nacional do Esporte (PNE), pensando que ambas compartilham de objetivos comuns, já que os esportes têm potencial de serem provedores da saúde biopsicossocial.

Ainda, o esporte como uma estratégia de inclusão social, por ser uma medida educativa e de lazer, pode ser utilizado para disseminar valores morais, como amizade, solidariedade, convívio harmônico e a não violência, desenvolvimento de cidadania e da socialização ${ }^{11}$. Estudo mostrou que o esporte melhora também o relacionamento com a família e o desempenho escolar, tornando os jovens com mais responsabilidade, organização, amadurecimento, deixando-os menos tímidos e com mais autoestima; diminuição de comportamento agressivo e rebelde, o que, consequentemente, melhora seu convívio social, tornando-os menos propensos a criminalidade ${ }^{12}$.

Assim, o esporte, sendo para a população uma das abordagens terapêuticas lúdicas e educativas com evidências de sua eficácia para os benefícios do corpo e mente, deve ser inserido nas Redes de Atenção à Saúde e nos programas nas escolas; para promover saúde e, consequentemente, reduzir os custos dos tratamentos em níveis mais altos de complexidade ${ }^{13,14}$.

Diante de todos os efeitos positivos que o esporte proporciona à saúde, percebe-se a importância do esporte de competição ou somente recreativo como promotor de saúde e a relevância de ações voltadas para estimular as práticas esportivas e o incentivo que a PNPS deve à PNE.

\section{POLÍTICA NACIONAL DO ESPORTE}

No Brasil, o movimento "Esporte para todos" foi o marco para o início da discussão do esporte como um direito social. Esse movimento teve influência internacional, que já discutia o esporte como uma estratégia para o desenvolvimento humano. A Constituição Federal de 1988, por meio do art. 217, afirma que é dever do Estado fomentar práticas desportivas formais e não formais ${ }^{15}$. No entanto, isso não entra em vigor na época, pois a criação do Ministério do Esporte aconteceu somente em 1995, mostrando que, apesar de perceber o esporte como estratégia de desenvolvimento, sua implementação não foi prioridade $^{16}$.

A PNE foi institucionalizada pela Resolução $n^{\circ} 5$, de 14 de junho de $2005^{17}$, aprovada pelo Conselho Nacional do Esporte, sendo alvo de vários debates na I Conferência Nacional do Esporte (CNE) em 2004.

Como objetivos da PNE, constam: a democratização e a universalização do acesso ao esporte e ao lazer, na perspectiva da melhoria da qualidade de vida da população brasileira; a promoção da construção e fortalecimento da cidadania, assegurando o acesso às práticas esportivas e ao conhecimento científico-tecnológico a elas inerente; a descentralização da gestão das políticas públicas de esporte e lazer; o fomento às práticas do esporte de caráter educativo e participativo para toda a população, além do fortalecimento da identidade cultural esportiva a partir de políticas e ações integradas com outros segmentos; o incentivo ao desenvolvimento de talentos esportivos em potencial e aprimoramento do desempenho de atletas e para-atletas de rendimento, promovendo a democratização dessa manifestação esportiva ${ }^{18}$. 
A PNE tem em sua base princípios que norteiam a reversão do quadro atual de injustiça, exclusão e vulnerabilidade social; o esporte e o lazer como direito de cada um e dever do Estado; a universalização e a inclusão social; e a democratização da gestão e da participação. Entre as diretrizes da PNE, estão: universalização do acesso e promoção da inclusão social; desenvolvimento humano; ciência e tecnologia do esporte; promoção da saúde; paz e desenvolvimento da ação; desenvolvimento econômico; gestão democrática; e descentralização da política esportiva e de lazer .

Entre as estratégias previstas na PNE, estão ${ }^{18}$ :

$\checkmark$ ampliação do acesso ao esporte em cada região do país como direito social;

$\checkmark$ garantia da oferta regular e da difusão da cultura das práticas esportivas escolares e de lazer para todas as pessoas (sem distinção de cor, raça, etnia, sexo, idade ou condição social);

$\checkmark$ ampliação e modernização de infraestrutura esportiva, contemplando a diversidade das práticas;

$\checkmark$ estruturação da Política Nacional de Recursos Humanos, articulada com estados e municípios, para capacitação e formação com caráter multiprofissional e multidisciplinar, em parceria com o MEC e IES, de Recursos Humanos atuantes em atividades esportivas em todos os níveis, de forma a atender o Sistema Nacional de Esporte e Lazer;

$\checkmark$ desenvolvimento do conhecimento, da ciência e da tecnologia do esporte;

$\checkmark$ oferecimento regular de práticas esportivas educacionais, de lazer e de alto rendimento, especialmente voltadas para a população em situação de vulnerabilidade social;

$\checkmark$ fortalecimento da participação da mulher no esporte;

$\checkmark$ promoção de práticas esportivas que atendam pessoas deficientes e com necessidades especiais; $\checkmark$ afirmação do esporte como meio de promoção da saúde em ações interdisciplinares com o Ministério da Saúde (MS), a Organização das Nações Unidas (ONU), o Conselho Nacional de Segurança Alimentar e Nutricional (Consea), entre outros, incluindo os órgãos congêneres nos estados e municípios;

$\checkmark$ implementação de ações de combate ao doping esportivo, bem como de prevenção ao seu uso, respeitando as normas e regulamentos dos organismos internacionais de combate ao doping;

$\checkmark$ criação de sistema integrado de diagnóstico, avaliação e documentação esportiva, amplo e aprofundado;

$\checkmark$ garantia da democratização da informação no âmbito esportivo;apoio e fortalecimento da gestão pública do esporte em todos os níveis;

$\checkmark$ estruturação do Sistema Nacional de Esporte e Lazer no País, compreendendo as esferas de atuação pública e privada, consolidando uma rede de gestores federais, estaduais e municipais, as entidades de prática e de administração esportiva, entidades representativas do esporte e do lazer, escolas públicas e privadas, universidades, prestadores de serviço e profissionais, técnicos, atletas e a população atendida;

$\checkmark$ estruturação de uma política de financiamento que esteja vinculada ao Sistema Nacional de Esporte e Lazer e que mobilize, articule, amplie e dinamize recursos para essa área, coordenando as iniciativas do setor público, em todas as esferas e do privado, assentada sob princípios sólidos de controle público;

$\checkmark$ transparência; modernização e fortalecimento das entidades de administração e da prática do esporte com vistas à democratização das entidades e à profissionalização da gestão;

$\checkmark$ ampliação da participação da comunidade esportiva e da sociedade na implementação da política, mantendo canais permanentes de comunicação e garantia de mecanismos de controle e avaliação 
permanentes da PNE, com a participação dos Conselhos e dos Gestores estaduais e municipais, e por meio da Conferência, visando ao acompanhamento contínuo das ações em todos os níveis de execução, para direcionar e redirecionar seu processo de planejamento.

A criação dessa política aponta o esporte e o lazer como fatores de promoção da saúde, abrangendo todas as esferas da população, sendo, portanto, um fator importante no que diz respeito à geração de emprego e renda, além de contribuir para o contexto social.

\section{POLÍTICA NACIONAL DE PROMO- ÇÃO DA SAÚdE X POLÍTICA NACIO- NAL DO ESPORTE: O DESAFIO DA INTERSETORIALIDADE}

Ao considerar a ampla causalidade que envolve o processo saúde-doença, devem-se expandir as atuações intersetoriais no campo das políticas de saúde, visando à melhoria das condições de vida e saúde ${ }^{19}$. Nesse sentido, os setores da educação e da ação social parecem ser os parceiros mais comuns nas ações intersetoriais das Políticas Públicas de Promoção da Saúde, apesar da evidente centralização no setor saúde ${ }^{20}$.

Como parte do enfoque de promoção de estilos de vida saudáveis, o Programa de Estímulo e Promoção da AF aproximou o MS dos Ministérios das Cidades, do Esporte, da Educação e da Cultura, e envolveu diferentes universidades e estados na Rede Nacional de Promoção da Saúde em vários municípios, na manutenção dos projetos financiados ${ }^{21}$.

No entanto, embora a criação da PNE e seus preceitos sejam essenciais para a população brasileira, há várias lacunas ainda a serem preenchidas. Os gestores municipais, estaduais e federais devem entender a importância dessa política como uma diretriz norteadora das etapas de planejamento e implementação desta na esfera municipal, principalmente no que diz respeito à melhora da comunicação do Ministério do Esporte com as secretarias municipais. É necessário aumentar a geração de demanda por parte do município; e, para isso, o conhecimento é indispensável, ou seja, é preciso ampliar o capital cultural, juntamente com o saber técnico e o engajamento político ${ }^{13,22}$.

Ainda, as dificuldades com relação à efetividade das ações da PNE se devem, em parte, ao despreparo dos profissionais de saúde em orientar a prática esportiva, enfatizando a importância da atuação do profissional de educação física nas Unidades Básicas de Saúde ${ }^{16}$.

A atuação da PNPS vai a esse encontro, no sentido de apoiar os profissionais de educação física e capacitar os profissionais de saúde quanto à orientação dos usuários a praticarem $\mathrm{AF}$ regularmente, já que o desconhecimento da população aos benefícios gerados pelo esporte ou exercício físico seria solucionado com ampla divulgação dos resultados das pesquisas científicas para a população em uma linguagem fácil e atrativa, no desenvolvimento de campanhas educativas à população sobre o esporte, ou apoiando mais fortemente as ações da PNE. Outro ponto positivo seria a diminuição do desperdício de recursos, que seriam reduzidos com a melhor elaboração dos projetos, como com o compartilhamento desses com as demais esferas do governo, novamente mostrando a importância da articulação entre essas políticas públicas.

Nesse sentido, a Estratégia Saúde da Família (ESF) e o Núcleo Ampliado de Saúde da Família (Nasf) devem, para além da interdisciplinaridade das ações entre os membros de suas equipes, promover a integração dos diversos setores sociais, entre eles, a prática regular de AF orientada, para compor ações estratégicas para o fortalecimento das ações em saúde ${ }^{22}$.

Apesar de já ter sido iniciado o processo 
de capacitação de gestores em promoção da saúde, muitos docentes da área da saúde e profissionais atuantes no SUS e na Saúde Pública/Coletiva ainda não tiveram a oportunidade de reciclar conhecimentos e ampliar suas visões de saúde, persistindo o enfoque preventivista e a visão biomédica que embasam ações setoriais ${ }^{17}$.

Outra necessidade refere-se ao desenvolvimento técnico-científico no esporte, principalmente no que diz respeito à melhoria das estratégias de implementação das ações. Isso se deve à inevitabilidade de políticas públicas mais realistas para o País. É preciso incentivar estudos em relação ao desenvolvimento de diagnóstico e avaliação das ações objetivando gerar conhecimentos que auxilie o aprimoramento das políticas públicas ${ }^{23,24}$.

Além disso, as propostas idealizadas pela PNE relacionadas com a inclusão social devem ser analisadas no âmbito de gastos governamentais. As três Conferências Nacionais do Esporte, realizadas em 2004, 2006 e 2010, destacaram a importância de ampliar o acesso ao esporte e lazer atendendo a grupos com diferentes necessidades e interesses, ou seja, não somente ao esporte de alto rendimento. Apesar dessas premissas serem discutidas nessas conferências com frequência, o financiamento do esporte de alto rendimento é constantemente maior do que para o esporte educacional e de participação. Como exemplo, é visto no orçamento governamental que o "Programa Esporte e Lazer na Cidade", que contempla especificamente as diferentes manifestações da população, é justamente o programa que conta com menos investimentos do governo federal, fazendo-se necessária a inversão de prioridades nas ações realizadas pelo governo no âmbito esportivo ${ }^{24-26}$. Ademais, sabe-se que, para se alcançar maior número de indivíduos em esportes de alto rendimento, é nas categorias de base, nas iniciantes, que se deve iniciar o trabalho, a fim de nortear o foco para o esporte como profissão.

Onde há projetos de inclusão social promovidos pelo esporte, verifica-se que maior incidência de indivíduos que participam desses projetos é na faixa etária de 10 anos a 13 anos; e, à medida que a faixa etária aumenta, a evasão se torna maior ${ }^{27}$, o que mostra que as ações ainda têm falta de atratividade, principalmente entre os adolescentes. Com isso, a medida seria atender mais às expectativas dos participantes, mas, principalmente, fortalecer as ações por meio de interações entre as políticas públicas. É importante também o trabalho com os cuidadores das crianças e adolescentes no que tange a identificar a importância que a prática de AF regular tem para futuras gerações.

Assim, destaca-se a importância de campanhas para motivar a prática esportiva e conscientizar profissionais da saúde e a população sobre seus benefícios, além de difundi-la em outros segmentos, buscando parcerias com outros setores públicos, saúde, educação. Caberia ao Estado fazer do esporte um mecanismo a ser operado juntamente com outros ministérios; e, nesse caso, principalmente com o MS e com a PNPS, dada a importância que o esporte tem em promover saúde. Essa integração entre as políticas, compartilhando recursos físicos e humanos, e reunindo subsídios, facilitaria o alcance das metas em comum. No entanto, o que se tem hoje é que essa comunicação entre os diversos setores do governo ainda é falha ${ }^{13}$.

Ainda, é de grande importância a atuação de profissionais, em especial os de educação física e os fisioterapeutas, com a função de orientar a população para um estilo de vida saudável com ênfase na realização das atividades físicas e esportivas, contribuindo para uma maior reflexão das pessoas e possíveis mudanças de hábitos que impliquem efetivamente sua qualidade de vida.

Considera-se ainda a prática de $\mathrm{AF}$ e esportiva regular uma ferramenta de baixo custo e de fácil acesso em comparação à gama de benefícios que produz. Entretanto, percebe-se que ainda prevalece uma necessidade de mais investimento em profissionais, especialmente o de educação física e 
o fisioterapeuta, aos quais cabe refletir sobre esses programas e políticas que procurem esclarecer melhor as pessoas sobre os benefícios das atividades físicas e esportivas de com vistas à busca de uma melhor qualidade de vida, proporcionando ao indivíduo, mediante compreensão do seu corpo, melhores condições de se manifestar por meio do movimento corporal.

\section{REFERÊNCIAS}

1. Katzmarzyk PT, Janssen I. The economic costs associated with physical inactivity and obesity in Canada: an update. Canadian Journal of Applied Physiology 2004; 29(1):90-115.

2. Matsudo SM. Atividade Física na promoção da saúde e qualidade de vida no envelhecimento. Rev Bras Educ Fís 2006; 20(5):135-7.

3. Nóbrega ACL, Freitas EV, Oliveira MAB, Leitão MB, Lazzoli JK, Nahas RM, et al. Posicionamento oficial da Sociedade Brasileira de Medicina do Esporte e da Sociedade Brasileira de Geriatria e Gerontologia: Atividade Física e saúde no idoso. Rev Bras Med Esporte 1999; 5(6):207-11.

4. Scabar TG, Pelicioni AF, Pelicioni MCF. Atuação do profissional de Educação Física no Sistema Único de Saúde: uma análise a partir da Política Nacional de Promoção da Saúde e das Diretrizes do Núcleo de Apoio à Saúde da Família - NASF. J Health Sci Inst 2012; 30(4):411-8.

5. Conselho Nacional de Educação. Parecer CNE/ CES no 584, de 3 outubro de 2018. Diretrizes Curriculares Nacionais do curso de graduação em Educação Física. Diário Oficial da União 2018, 3 out.

6. Hupin D, Roche F, Gremeaux V, Chatard JC, Oriol M, Gaspoz JM, et al. Even a low-dose of moderate-to-vigorous physical activity reduces mortality by $22 \%$ in adults aged $\geq 60$ years: a systematic review and meta-analysis. $\mathrm{Br} \mathrm{J}$ Sports Med 2015; 49(19):1262-7.

7. Brasil. Ministério da Saúde. Secretaria de Vigilância em Saúde. Secretaria de Atenção à Saúde. Política Nacional de Promoção da Saúde: PNPS: Anexo I da Portaria de Consolidação $n^{\circ} 2$, de 28 de setembro de 2017, que consolida as normas sobre as políticas nacionais de saúde do SUS/ Ministério da Saúde, Secretaria de Vigilância em Saúde, Secretaria de Atenção à Saúde. - Brasília: Ministério da Saúde; 2018. 40 p.

8. Carvalho FFB, Cohen SC, Akerman M. Refletindo sobre o instituído na Promoção da Saúde para problematizar “dogmas”. Saúde Debate 2017; 41(spe3):265-76.

9. Brasil. Ministério da Saúde. Portaria $n^{\circ} 719$, de 7 de abril de 2011. Institui o Programa Academia da Saúde no âmbito do Sistema Único de Saúde. Diário Oficial da União 2011; 8 abr.

10. Brasil. Ministério da Saúde. Portaria n²681, de 7 de novembro de 2013. Redefine o Programa Academia da Saúde no âmbito do Sistema Único de Saúde (SUS). Diário Oficial da União 2013; 8 nov.

11. Azevedo MAO, Gomes Filho A. Competitividade e inclusão social por meio do esporte. Rev. Bras. Ciênc. Esporte, Florianópolis 2011; 33(3):589-603.

12. Sanches SM, Rubio K. A prática esportiva como ferramenta educacional: trabalhando valores e a resiliência. Educ e Pesqui 2011;37(4):825-41.

13. Alves JAB, Pieranti OP. O estado e a formulação de uma política nacional de esporte no Brasil. RAE 2007; 6(1).

14. Moretti AC, Almeida V, Westphal MF, Bógus CM. Práticas corporais/AF e políticas públicas de promoção da saúde. Saúde Soc 2009; 18(2):346-354. 
15. Brasil. Constituição (1988). Constituição da República Federativa do Brasil. Brasília, DF: Senado Federal: Centro Gráfico; 1988. 292 p.

16. Correia MM. Projetos sociais em educação física, esporte e lazer. Rev. Bras. Cienc. Esporte 2008; 29(3):91-105.

17. Azevedo E, Pelicioni MCF, Westphal MF. Práticas intersetoriais nas políticas públicas de promoção de saúde. Physis 2012; 22(4):1333-1356.

18. Brasil. Política Nacional do Esporte. Ministério do Esporte. Presidência da República, Casa Civil, Subchefia para Assuntos Jurídicos. Brasília, DF: ME, p 1-24; 2005.

19. Azevedo E, Pelicioni MCF, Westphal MF. Práticas intersetoriais nas políticas públicas de promoção de saúde. Physis 2012; 22(4):1333-1356.

20. Mendes R. Cidades saudáveis no Brasil e os processos participativos: os casos de Jundiaí e Maceió [doutorado]. São Paulo (SP): Faculdade de Saúde Pública, Universidade de São Paulo; 2000.

21. Brasil. Ministério da Saúde. Promoção da Saúde V. Brasília: OPAS; 2009 (Painel de Indicadores do SUS n. 6).

22. Giovanella L, Mendonça MHM, Almeida PF, Escorel S, Senna MCM, Fausto MCR, et al. Saúde da família: limites e possibilidades para uma abordagem integral de atenção primária à saúde no Brasil. Ciên Saúde Colet 2009; 14(3):783-794.

23. Terra AM, Brandão HC, Flausino MS, Mascarenhas F. As conferências nacionais do esporte: avanços e limites na construção de políticas de esporte e lazer. [internet]. Revista Digital 2009 [acessado 2019 jul 26]; 14(137). Disponível em: https://www.efdeportes.com/efd137/ construcao-das-politicas-de-esporte-e-lazer htm
24. Athayde P, Mascarenhas F, Salvador E. Primeiras aproximações de uma análise do financiamento da política nacional de esporte e lazer no Governo Lula. Rev Bras de Ciências do Esporte 2015; 37(1):2-10.

25. Anais do XVII Congresso Brasileiro de Ciências do Esporte e IV Congresso Internacional de Ciêcias do Esporte. 11 - 16 de set de 2011; Porto Alegre (RS).

26. Ramos G, Amaro DA, Amaral JMV, Barboza MRS, Maciel RM, Silva LAM. Atividade física, esporte e a qualidade de vida. Revista Saúde e Educação, Coromandel 2017; 2(2):29-48.

27. Vianna JA, Lovisolo HR. Projetos de inclusão social através do esporte: notas sobre a avaliação. Rev Mov 2009; 15(3):145-162.

Recebido: 17/09/2019 Aprovado: 06/05/2020 